\title{
MICROPATTERNED HIGH DENSITY NANOWIRES ON PAPER SUBSTRATES
}

\author{
P. Mostafalu, S. Sonkusale \\ NanoLab, Department of Electrical and Computer Engineering, Tufts University \\ Medford, MA, USA
}

\begin{abstract}
In his study we present a novel approach for fabrication of high-density nanowires on paper substrates for improved paperbased electrodes, sensors and electronic devices. Different types of nanowires including platinum, nickel and copper are fabricated and patterned on paper. Nanowires are characterized with scanning electron microscopy and energy dispersive spectroscopy. Nanowires on paper were utilized as a dry electrode with excellent electrode-tissue impedance suitable for the recording of electrocardiogram signals.
\end{abstract}

\section{INTRODUCTION}

Development of low cost reliable environmentally sustainable medical diagnostic devices is receiving increased attention. [1, 2] Accordingly, paper has been suggested as a suitable substrate material for such devices. Paper is a flexible, lightweight and biocompatible platform which has been endorsed for sensing and electronics applications, e.g. capacitive touch pads, glucose sensors and bioassays.[3, 4] Moreover, fabrication of paper-based devices is straightforward and requires low cost, room temperature processing steps depending on the type of the paper used.[3-5]

Nanowires and nanoparticles, with their unique properties such as high surface area and outstanding electrical transport behavior, are emerging materials for implementation of electrodes for sensors, batteries and supercapacitors.[6, 7] Biomedical devices have also benefited from the use of nanomaterials with applications ranging from the recording of neural activity, to the detection of biological targets such as DNA and dopamine using nanowires, nanopores and nanoneedles.[8, 9]

Introducing nanowires and nanopowders on paper substrates will synergistically bring together the low cost paradigm of paperbased substrates with the outstanding physical, electrical and chemical performances of nanomaterials, providing a key enabler to realize ideal electrodes for many applications. Generally, numerous techniques have been reported for the growth of nanowires. Vapor-Liquid-Solid (VLS) and Chemical-VaporDeposition (CVD) are two standard methods for nanowires growth but these methods require high temperatures (more than $300^{\circ} \mathrm{C}$ ) which are not feasible owing to low ignition temperature of paper.[10, 11] Consequently, the template-assisted mechanism offers a solution to this problem, which stimulates nanowire growth with a low temperature process that is compatible with paper-based devices. In the template-assisted method, a porous membrane is employed to electrodeposit nanowires through its pores. For the choice of membranes, two kinds have been already proposed: Anodized Aluminum Oxide (AAO) and Poly Carbonate Membrane (PCM). AAO process is simpler and more straightforward, and is used in this study. To make a template, first, a $\sim 50 \mu \mathrm{m}$ thick ultra-pure layer of aluminum film is required. But, difficulty in deposition of such a thick layer and its anodization make direct growth of nanowire on paper unworkable. So we have developed an approach, which involves making an AAO template separately, and then transferring the template on paper for subsequent growth of nanomaterials through them.

In this study we also present a low cost approach not just for the fabrication of metal nanowires on paper substrates but also on their patterning and alignment. The fabrication process is simple and straightforward; screen printing method is used for patterning of electrodes, and double-sided tape engaged for initial attachment. The method is simple and requires no microfabrication facilities, and as such could be applied on other unconventional substrates other than papers.

To demonstrate a viable application for such a nanowire on paper-based electrodes, we utilize them as dry flexible electrode for the recording of electrocardiogram (ECG) signals. Typically, wet gel adhesives are needed for ECG recording, which make them inconvenient for long term monitoring. However, the high surface area and low impedance of the proposed paper based electrodes facilitates ease of recording even with a dry attachment to the skin. While biopotential recording is the only example explored in this study, such nanowire electrodes on paper will find use as electrodes for stimulation and recording from electrogenic cells, electrochemical and biological sensors, supercapacitors, and batteries.

\section{MATERIALS AND METHODS}

\section{Chemical and materials}

In this study Whatman chromatography paper grade 1 (Sigma-Aldrich) Reynolds parchment paper and photo paper was used as a substrate. Whatman AAO membranes with different pore sizes (20 nm and $200 \mathrm{~nm}$ ) were used as templates. Thin, solvent resistant double-sided tape (Mcmaster, MA) was utilized for attachment of AAO membrane to the paper. Silver ink (Ag 510, Conductive Compound, MA) was used as a material for screen printing of the electrodes. Copper, nickel and platinum electroplating solutions (Technic Inc, USA) were purchased and used without further modification.

\section{Fabrication process}

First, the double-sided tape was patterned as a mask by laser engraving using a laser cutter (Versa VLS2.40) (power 40\%, speed $20 \%$ ) and it was attached to the paper (photo or parchment) which served as a substrate. Then silver ink was spin coated (5000 rpm for $60 \mathrm{~s})$ on the top and was cured $\left(15\right.$ minute in $\left.121^{\circ} \mathrm{C}\right)$ and left in the desiccator (30 minutes) to remove all remaining residues. At the end of this step, interconnects and electrode pads on the paper were prepared. For electrodeposition of nanowire through pores of the AAO membrane, a conductive seed layer is required on top of the AAO. Therefore small circular electrodes (200 nm diameter) were patterned on the top-side of AAO template. To create these circular electrodes, the Mylar shadow mask is prepared by laser cutter and attached to the AAO template using Aerosol spray which is easy to remove adhesive; then either $200 \mathrm{~nm}$ of the silver was sputtered (NSC 3000 DC Magnetron Sputter tool) or gallium indium eutectic (Sigma Aldrich USA) was painted. In the next step, patterned interconnects on the paper were covered with a tape and a conductive epoxy (Norland NCA130) was screen printed on the patterned paper-based electrodes. Cover of the double-sided tape was peeled off and the AAO template with patterned electrodes was then attached on the paper; the epoxy was cured using UV light and heat $\left(2\right.$ hours, $\left.80^{\circ} \mathrm{C}\right)$ to completely cure and provide a good electrical connection. The size of patterned electrodes on the paper is larger than size of electrodes on the AAO to provide simple alignment.

The electrodeposition was performed by chronopotentiometry method with the current density of $1 \mathrm{~mA} / \mathrm{cm}^{2}$ using Potentiostat (PGSTATN12, Autolab, Mterohm) for several hours with exact 


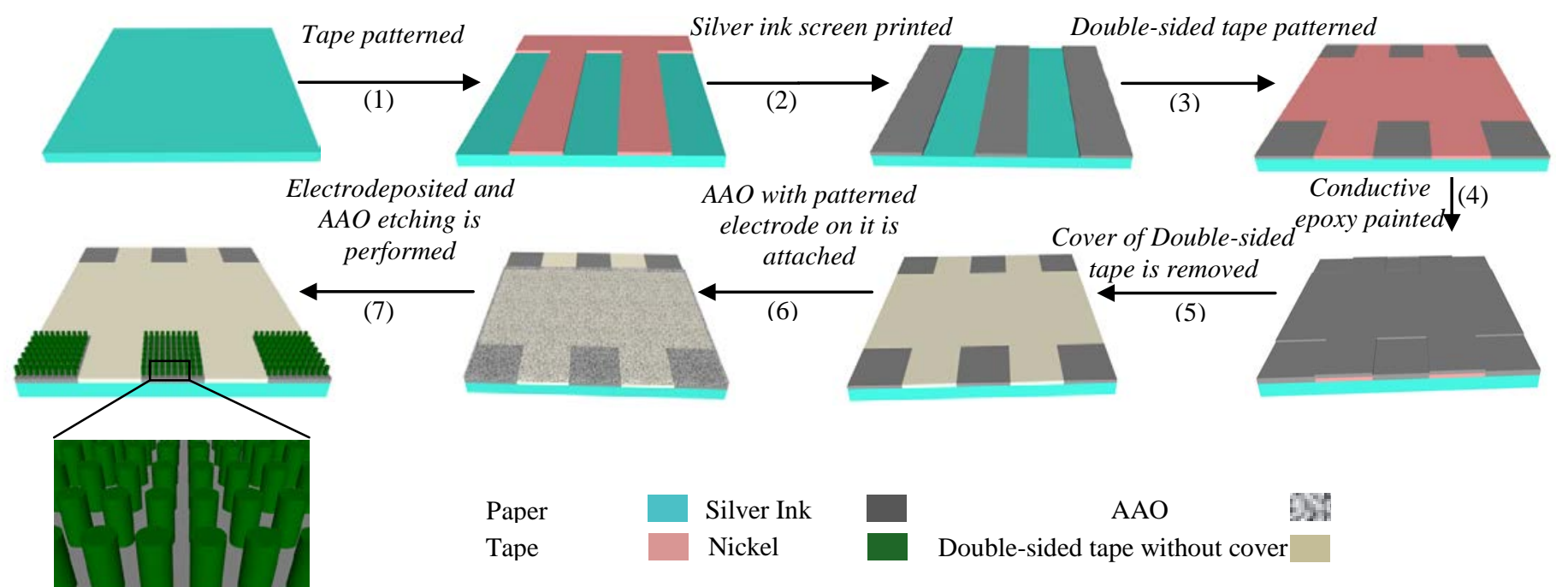

Figure 1: Fabrication process of nanowire on paper.

time depending on the desired length of nanowires. After electrodeposition, the AAO template was etched in sodium hydroxide (2M) for 1 hour. For the electrodeposition and etching the hand-made electrochemical cell has been used. Figure 2 shows patterned nanowires electrodes on the paper.

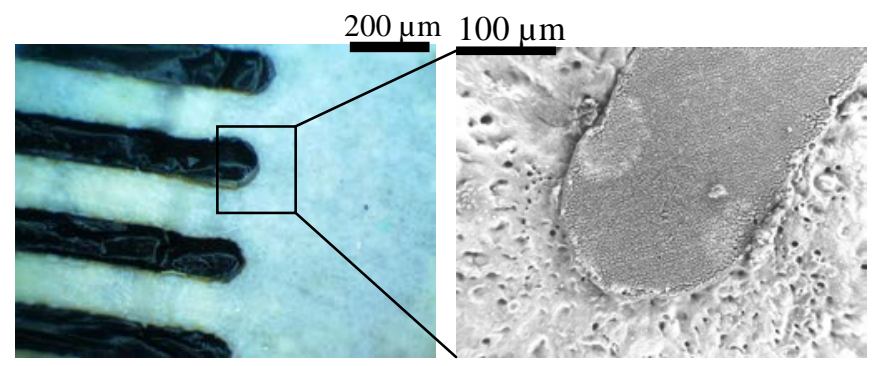

Figure 2: Patterned electrode on the paper after growth of nanowire.

\section{RESULT AND DISCUSSION}

The growth of nanowires with the desired length and diameter is one of the important design parameters. In general, the length of nanowires in the template-assisted approach is controlled by varying the time of deposition, current density, thickness of the AAO template, nature and type of the electrodeposited materials and the concentration of elctrodeposition bath. Current density affects the quality of deposition; it always should be kept as low as possible (less than $5 \mathrm{~mA} / \mathrm{cm}^{2}$ ) to get a uniform electrodeposition. Here we have used a current density of $1 \mathrm{~mA} / \mathrm{cm}^{2}$, which gives a deposition rate of $20 \mathrm{~nm} /$ minute. The thickness of Whatman AAO templates are $60 \mu \mathrm{m}$ so the maximum length of nanowire is limited to $60 \mu \mathrm{m}$. Electrodeposition time is the most effective parameter in the length of nanowires; the longer time would cause longer nanowires. For example we have performed copper electrodeposition for 1 hour, 2 hours and 5 hours, and nanowires with lengths of 2, 5, $10 \mu \mathrm{m}$ were achieved respectively. Moreover, another parameter that controls the length and morphology of the

nanowires is the type of material itself. Voltage during deposition of the copper is kept very small around $0.2 \mathrm{~V}$; for nickel it is around $0.6 \mathrm{~V}$ and for platinum it is high around $1.5 \mathrm{~V}$. These numbers were arrived at empirically and the differences could be because of the different resistivity of the plating solutions with copper solution being the least resistive.

The second parameter in growth of nanowires is diameter which is limited to the pore size of AAO template. In this study, we used a commercial Whatman AAO template with pore size of $20 \mathrm{~nm}$ and $200 \mathrm{~nm}$. The SEM images in Figure 3a and Figure3b show nickel nanowire with these two different diameters.

(a)

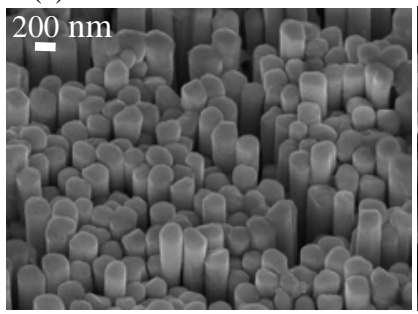

(c)

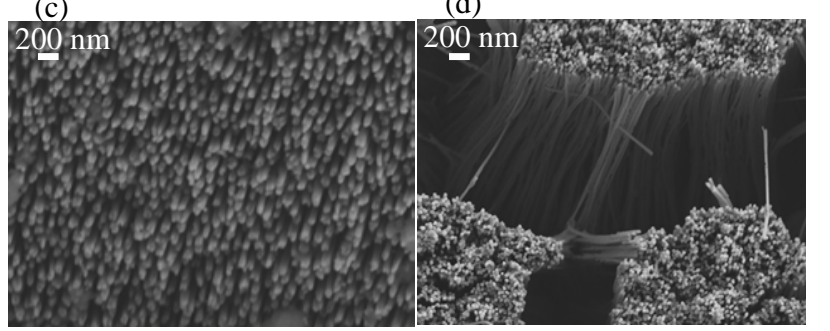

Figure 3: SEM images of paper-based nanowires aand 20nm (a) Nickel nanowires with diameter of $200 \mathrm{~nm}$ (b) Nickel nanowires with diameter of $20 \mathrm{~nm}$ (c) Platinum nanowires with diameter of $20 \mathrm{~nm}(d)$ Copper nanowires with diameter of $200 \mathrm{~nm}$.

However, electrodeposition of nanowires on the paper-based electrode has some other issues which should also be considered. Since papers are flexible materials, during electrodeposition different parts of electrodes might be a different distance from the 

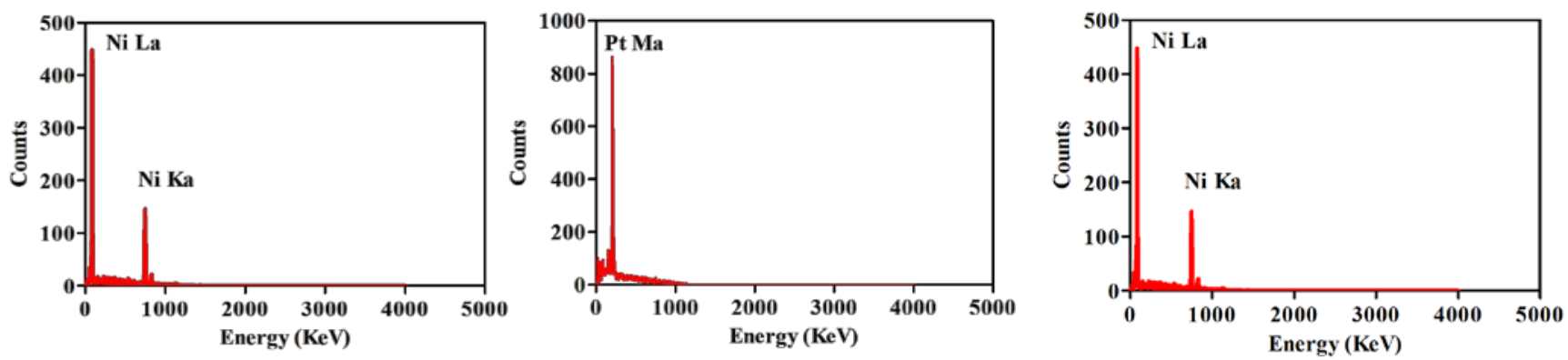

Figure 4: EDS analysis of different types of nanowire on paper (a) Copper nanowire (b) Platinum nanowire (c) Nickel nanowire.

anode and there could be non-uniformity in the length distribution. Utilizing double-sided tape and applied force with a clamp in the handmade cell made it more stable during deposition in our experiments. In this case, double-sided tape should be smooth and clamp tight enough to not let solution go through the pores of AAO template in the unpatterned areas. Leaching of the solution in the unpatterned area reduces the electrodeposition rate but will not stop the nanowire growth.

Another issue which may also be problematic is the unwanted soaking of the paper from the solution even in areas where there are no electrodes. Using hydrophobic paper like a parchment paper, wax paper or photography paper could solve this problem. Another solution is to using wax printing to form hydrophobic channel around the electrodes, which also limits the flow of the solution only in the hydrophillic areas.

\section{Electrode characterization}

For the physical characterization of nanowires, scanning electron microscopy (SEM) and Energy-dispersive X-ray spectroscopy (EDS) has been used. SEM images of nanowire electrodes were acquired using FESEM ultra55 (12 KV) to determine the structural features of the fabricated nanowires on paper substrate. Samples were mounted on the aluminum stubs using conductive carbon paint. As shown in Figure 2, we successfully grew nickel nanowires (Figure 3a) platinum nanowires (Figure 3c) and copper nanowires (Figure 3d). Figure 3a shows nickel nanowires with diameters of $200 \mathrm{~nm}$ and $20 \mathrm{~nm}$. Copper nanowires with length of more than $10 \mu \mathrm{m}$ are achieved (Figure 3d). We used Energy Dispersive Spectroscopy (EDS) for investigation of nanowires composition. The electron beam from SEM tools excited the sample and an X-ray spectrum emitted by the sample was obtained using the X-ray detector. Results of EDS analysis for nickel, copper and platinum high density nanowires are shown in Figure 4.

\section{Electrocardiography}

Cardiovascular diseases is the leading cause of the death. One could manage chronic heart conditions by continuous monitoring of electrocardiogram (ECG) activity. Development of an electrode and device for long term ECG monitoring is therefore essential to monitor heart activity in at-risk patients.

The electrodes that interface with the skin are very important part of the device to monitor heart's electrical activity. However current ECG electrodes that are typically used for this applications are made from $\mathrm{Ag} / \mathrm{AgCl}$ with a gel electrolyte interface. It dries over time when used for long time monitoring and also degrades considerably due to sweat and motion; this consequently affects the quality of the signal received from the heart.[12] Moreover wet electrodes are uncomfortable for the patients since direct skin contact is necessary at all times. Recently dry ECG electrodes have been reported as a promising approach for replacement of wet ECG electrodes because they can facilitate long term monitoring with little inconvenience to the patient. However dry electrodes are very sensitive to motion artifacts and poor contact with skin and limited biocompatibility restrict their utility. In this study we have proposed wearable and flexible paper-based nanowire electrode as a viable dry ECG electrode for long term ECG monitoring.

Circular ECG electrodes with a diameter of $10 \mathrm{~mm}$ were fabricated according to the fabrication process mentioned in Section 2.1. For the evaluation of the ECG electrode, we have used a 3 electrodes setup; two nanowire-based electrodes and one regular screen printed silver electrode on the paper were connected to left arm, right arm and left leg as it is shown in figure 5b. Since the electrical signal is very small, its amplification and filtration is required. The electrical circuit composed of mentioned components, is implemented on a printed circuit board (PCB) using the circuit topology discussed here. [13]

Flexibility and easy attachment of nanowire on paper electrode using a double sided-tape makes for a very easy to interface electrode system for these measurements.

As mentioned earlier, a critical aspect to receive a high fidelity ECG signal is a good contact between electrodes and skin. The electrode-skin contact impedance has been measured to characterize the proposed ECG electrode. For the measurement of the impedance interface, the configuration which is shown in Figure 5a was used; based on the approach proposed in previous studies.[14] In this set up, 3 electrodes A, B and C were attached to the skin. The electrode $\mathrm{B}$ is the main electrode which is addressable for the measuring of its contact impedance with skin; the current in the range of $1-20 \mu \mathrm{A}$ (using function generator and $\mathrm{R}_{\text {ext }}$ ) is applied to the electrode A while B and C are in open load. The voltage measured between electrodes $B$ and $C\left(V_{\text {out }}\right)$ will depend only on the drop across the tissue-electrode impedance for electrode $\mathrm{B}\left(\mathrm{R}_{\mathrm{e} 2}=\mathrm{V}_{\text {out }} / \mathrm{I}_{\mathrm{in}}\right)$. As figure $6 \mathrm{~b}$ shows impedance magnitude was changing for $100 \mathrm{~K} \Omega$ to $1 \mathrm{~K} \Omega$ when frequency varied from $5 \mathrm{~Hz}$ to $1.5 \mathrm{KHz}$.

\section{CONCLUSION}

In this study we have presented micropatterened nanowire electrode on paper substrates. Different types of metal nanowires have been grown and electrodes are characterized using SEM. Using this approach, we were able to fabricate nanowires with different lengths and diameters on flexible paper substrate. Element composition of materials was analyzed using EDS measurements. Finally electrodes were used as a viable dry alternative to wet gel electrodes for recording of the electrocardiogram signal. Impedance between skin and an electrode was measured; it varied from $100 \mathrm{~K} \Omega$ to $100 \Omega$ when 
(a)

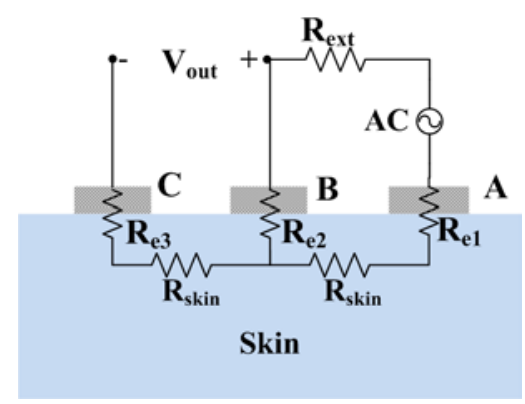

(b)

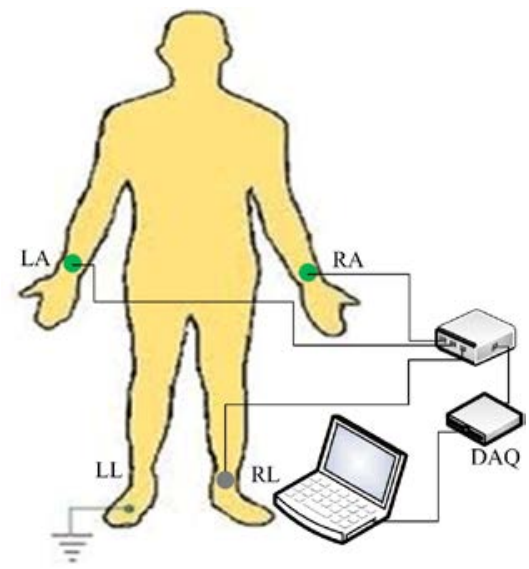

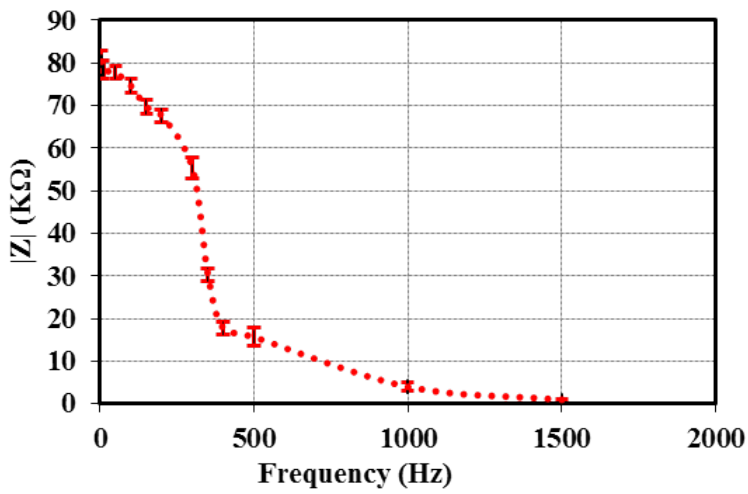

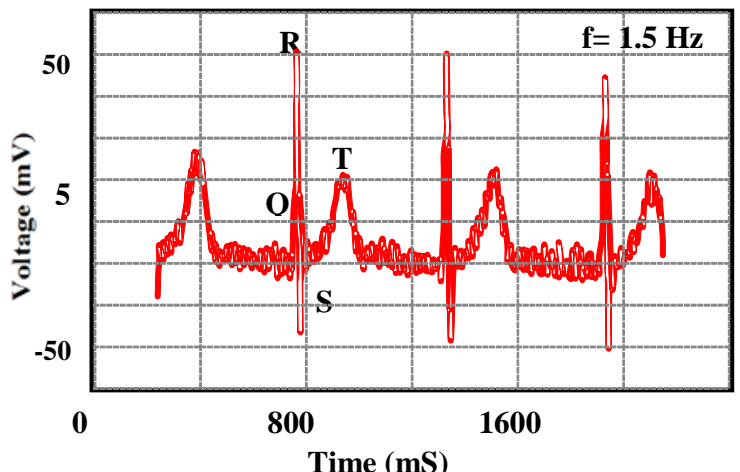

Figure 5: ECG analysis using PNE (a) Electrode-skin contact impedance measurement (b) methodology and recorded ECG signal.

frequency tuned from $5 \mathrm{~Hz}$ to $100 \mathrm{KHz}$ and is found to be comparable to the literature.

\section{ACKNOWLEDGMENT}

Device was fabricated in Tufts Micro and Nanofabrication Facility. Project was founded by National Science Foundation ECCS0955024 and EFRI-1240443. Travel support has been generously provided by the Transducer Research Foundation.

\section{REFERENCES}

[1] Z. Nie, C. A. Nijhuis, J. Gong, X. Chen, A. Kumachev, A. W. Martinez, et al., "Electrochemical sensing in paper-based microfluidic devices," Lab on a Chip, vol. 10, pp. 477-483, 2010.

[2] J. R. Windmiller and J. Wang, "Wearable Electrochemical Sensors and Biosensors: A Review," Electroanalysis, vol. 25, pp. 29-46, 2013.

[3] E. J. Maxwell, A. D. Mazzeo, and G. M. Whitesides, "Paper-based electroanalytical devices for accessible diagnostic testing," MRS Bulletin, vol. 38, pp. 309-314, 2013.

[4] A. D. Mazzeo, W. B. Kalb, L. Chan, M. G. Killian, J.-F. Bloch, B. A. Mazzeo, et al., "Paper-Based, Capacitive Touch Pads," Advanced Materials, vol. 24, pp. 28502856, 2012.

[5] G. Chitnis, Z. Ding, C.-L. Chang, C. A. Savran, and B. Ziaie, "Laser-treated hydrophobic paper: an inexpensive microfluidic platform," Lab on a Chip, vol. 11, pp. 11611165, 2011.

[6] P. Mostafalu and S. Sonkusale, "Paper-based supercapacitor using micro and nano particle deposition for paper-based diagnostics," in Sensors, 2013 IEEE, 2013, pp. 1-4.

[7] A. Vlad, A. L. M. Reddy, A. Ajayan, N. Singh, J.-F. Gohy, S. Melinte, et al., "Roll up nanowire battery from silicon chips," Proceedings of the National Academy of Sciences, vol. 109, pp. 15168-15173, September 18, 20122012.

[8] P. Xie, Q. Xiong, Y. Fang, Q. Qing, and C. M. Lieber, "Local electrical potential detection of DNA by nanowire-nanopore sensors," Nature Nanotech, vol. 7, 2012.

[9] R. Esfandyarpour, H. Esfandyarpour, J. S. Harris, and R. W. Davis, "Simulation and fabrication of a new novel 3D injectable biosensor for high throughput genomics and proteomics in a lab-on-a-chip device," Nanotechnology, vol. 24, pp. 465301-465311, 2013.

[10] R. S. Wagner and W. C. Ellis, "Vapor-Liquid-Ssolid mechanism of single crystal growth," Applied Physics Letters, vol. 4, pp. 89-90, 1964.

[11] D. Wang and H. Dai, "Low-Temperature Synthesis of Single-Crystal Germanium Nanowires by Chemical Vapor Deposition," Angewandte Chemie, vol. 114, pp. 4977-4980, 2002.

[12] J. Ha-Chul, M. Jin-Hee, B. Dong-Hyun, L. Jae-Hee, C. Yoon-Young, H. Joung-Sook, et al., "CNT/PDMS Composite Flexible Dry Electrodesfor Long-Term ECG Monitoring," IEEE Transactions on Biomedical Engineering,, vol. 59, pp. 1472-1479, 2012.

[13] T. Instruments, "Precision, Low Power Instrumetation Amplifier," vol. INA118 datasheet, Dec. 2011.

[14] M. S. SPACH, R. C. BARR, J. W. HAVSTAD, and E. C. LONG, "Skin-Electrode Impedance and Its Effect on Recording Cardiac Potentials," Circulation, vol. 34, pp. 649-656, 1966.

\section{CONTACT}

* S. Sonkusale, tel: +1-617-627-5113; sameer@ece.tufts.edu 